\title{
Predictors of Overweight and Obesity in American Indian Families With Young Children
}

\author{
Alexandra K. Adams, MD, PhD ${ }^{1}$; Emily J. Tomayko, PhD, RD ${ }^{2}$; Kate A. Cronin, $\mathrm{MPH}^{3}$; \\ Ronald J. Prince, $\mathrm{MS}^{4}$; Kyungmann Kim, $\mathrm{PhD}^{5}$; Lakeesha Carmichael, $\mathrm{MS}^{6}$; \\ Tassy Parker, PhD, $\mathrm{RN}^{7}$
}

\begin{abstract}
Objective: To describe sociodemographic factors and health behaviors among American Indian (AI) families with young children and determine predictors of adult and child weight status among these factors.

Design: Descriptive, cross-sectional baseline data.

Setting: One urban area and 4 rural AI reservations nationwide.

Participants: A total of 450 AI families with children aged 2-5 years participating in the Healthy Children, Strong Families 2 intervention.

Intervention: Baseline data from a healthy lifestyles intervention.

Main Outcome Measures: Child body mass index (BMI) z-score and adult BMI, and multiple healthy lifestyle outcomes.

Analysis: Descriptive statistics and stepwise regression.

Results: Adult and child combined overweight and obesity rates were high: $82 \%$ and $40 \%$, respectively. Food insecurity was high (61\%). Multiple lifestyle behaviors, including fruit and vegetable and sugar-sweetened beverage consumption, adult physical activity, and child screen time, did not meet national recommendations. Adult sleep was adequate but children had low overnight sleep duration of $10 \mathrm{~h} / \mathrm{d}$. Significant predictors of child obesity included more adults in the household $(P=.003 ; \beta=0.153)$, an adult AI caregiver $(P=.02 ; \beta=0.116)$, high adult BMI $(P=.001 ; \beta=0.176)$, gestational diabetes, high child birth weight $(P<.001 ; \beta=0.247)$, and the family activity and nutrition score $(P=.04 ; \beta=0.130)$.

Conclusions and Implications: We found multiple child-, adult-, and household-level factors influence early childhood obesity in AI children, highlighting the need for interventions to mitigate the modifiable factors identified in this study, including early life influences, home environments, and health behaviors.

Key Words: health behavior, healthy lifestyle, North American Indians, pediatric obesity (J Nutr Educ Behav. 2019; 51:190-198.)
\end{abstract}

Accepted July 16, 2018. Published online September 18, 2018.

\footnotetext{
${ }^{1}$ Center for American Indian and Rural Health Equity, Montana State University, Bozeman, MT

${ }^{2}$ School of Biological and Population Health Sciences, College of Public Health and Human Sciences, Oregon State University, Corvallis, OR

${ }^{3}$ Department of Surgery, School of Medicine and Public Health, University of Wisconsin, Madison, WI

${ }^{4}$ Department of Population Health, School of Medicine and Public Health, University of Wisconsin, Madison, WI

${ }^{5}$ Department of Biostatistics and Medical Informatics, School of Medicine and Public Health, University of Wisconsin, Madison, WI

${ }^{6}$ Frontier Science and Technology Research Foundation, Madison, WI

${ }^{7}$ Department of Family and Community Medicine, School of Medicine, University of New Mexico, Albuquerque, NM

Conflict of Interest Disclosure: The authors have not stated any conflicts of interest.

Address for correspondence: Alexandra K. Adams, MD, PhD, Center for American Indian and Rural Health Equity, Montana State University, AJM Johnson Hall 103B, PO Box 173485, Bozeman, MT, 59715; E-mail: alexandra.adams2@montana.edu

(C) 2018 The Authors. Published by Elsevier Inc. on behalf of Society for Nutrition Education and Behavior. This is an open access article under the CC BY-NC-ND license. (http:// creativecommons.org/licenses/by-nc-nd/4.0/)

https://doi.org/10.1016/j.jneb.2018.07.011
}

\section{INTRODUCTION}

Obesity rates in American Indian (AI) children are among the highest of all races and ethnicities. ${ }^{1}$ Moreover, AI children are the only group of children for whom an increase in obesity prevalence has been reported since 2003. ${ }^{2}$ Development of obesity in young AI children is of particular concern not only because it persists into later life and greatly increases the risk for chronic disease but also because of other health risk factors unique to these families, including historical trauma, extremely high rates of poverty, and inconsistent access to health care. ${ }^{3,4}$ Despite evidence for the importance of early years in determining individual factors related to obesity risk, little is known about these risk factors 
among AI children, who experience disproportionately high risk for the development of obesity. Specifically, there is scant literature on obesity and related health behaviors in very young AI children (aged $2-5$ years) and no studies to date have assessed this age group concurrently with information about an adult caregiver or included data on health behaviors of this age group within the family home environment.

The Healthy Children, Strong Families 2 intervention was designed to target this critical window by focusing on early childhood and the family home environment in AI communities. Healthy Children, Strong Families 2 consisted of monthly mailed toolkits delivering lessons focusing on child obesity prevention and health promotion coupled with text messages and Facebook; it is described in detail elsewhere. ${ }^{5,6}$ The current article presents cross-sectional child-, adult-, and family-level data at baseline enrollment into Healthy Children, Strong Families 2, which offer a critical opportunity to assess obesity and related risk factors among AI families with young children. The dataset includes 450 adult-child dyads from 4 rural and 1 urban AI community ranging in population density from approximately 3.5 to 3,000 people per square mile. This geographic diversity is noteworthy because few studies included urban AI families. The study also explored relationships among multiple factors influencing child body mass index (BMI) z-score and adult BMI within these households, which may provide important insights into future intervention design with these communities.

\section{METHODS}

\section{Study Design and Participants}

This study describes cross-sectional baseline data from Healthy Children, Strong Families 2, a randomized controlled trial of an obesity prevention intervention. (The methods are described in full elsewhere. ${ }^{6}$ ) Community stakeholders were engaged in all phases of the study, from participant recruitment through discussion and contextualizing of study findings. Enrollment occurred in 5 communities from February, 2013 to April, 2015. Informational brochures were sent home with children at Head Start programs for the reservationbased sites; information was displayed at a health center for the urban site. Inclusion criteria consisted of enrolling a child aged 2-5 years, the ability to attend data collection visits, and a working cell phone (because of the delivery of some intervention components via text messaging). Identifying as AI was not required because race, ethnicity, and culture are often complicated issues among families in these communities. For example, a parent may not identify as AI but will identify the child as AI or vice versa, leading to perceived discrepancies in numbers of AI participants in the current study. However, all families were recruited either from within reservations or from organizations providing services to AI families. The researchers obtained institutional review board approvals from the University of Wisconsin, participating tribal councils, and tribal institutional review boards upon request. Adult participants provided signed informed consent for themselves and their participating child.

\section{Anthropometrics and Surveys}

Height and weight were collected according to standardized protocols and converted to age- and sex-specific BMI percentiles for children ${ }^{7}$ and BMI for adults. ${ }^{8}$ Adult participants who were pregnant provided self-reported prepregnancy weight. Participating adults also completed a packet of validated self-report surveys. Screeners based on the National Health and Nutrition Examination Survey 2009-2010 (adults) and the 2010 National Youth Physical Activity and Nutrition Survey ${ }^{10}$ (children) assessed foods eaten during the previous week; the Godin Leisure-Time Exercise Questionnaire $^{11}$ (adults) and the Netherlands Physical Activity Questionnaire $^{12}$ (children) assessed physical activity; the Family Nutrition and Physical Activity Survey ${ }^{13}$ assessed family activity and the home environment (with 2 validated questions on food security from the US Department of Agriculture
Household Food Security Survey ${ }^{14}$ ); the Physical Activity and Nutrition Self-Efficacy Scale, ${ }^{15}$ Perceived Stress Scale, ${ }^{16}$ and 12-Item Short Form Health Survey $(\text { SF-12 })^{17}$ assessed stress and other psychosocial measures; and a validated survey assessed cultural identity. ${ }^{18}$ The research group created or adapted surveys to assess health history, sleep, screen time use, social media use (adult only), and readiness to change in 6 health behaviors (adult only, assessed on a 5-point scale, with higher scores indicating greater readiness).

\section{Analysis}

Descriptive statistics were performed on physical measures and surveys. The study also examined the possible association of sociodemographic variables and health behaviors and attitudes with adult BMI and child BMI z-score using a stepwise multiple regression approach (version 23, SPSS Statistics, IBM Corporation, Armonk, NY; 2015), with the demographic and health behavior variables at baseline entered as predictors. The stepwise procedure selected a subset to include in the final model that explained the maximal variance. ${ }^{19,20}$ For adults, the following variables were included: baseline Family Nutrition and Physical Activity scale, SF-12, Physical Activity and Nutrition Self-Efficacy scale, Perceived Stress Scale, and cultural involvement scale scores; household income, food security status, and urban vs rural designation; adult education; adult screen time, sugar and fruit/vegetable intake, and sleep; and adult ethnicity. Child predictors were entered in three stages: first, "Sociodemographic Variables" (number of adults/children in household; household income, food security status, and urban vs rural designation; adult education, ethnicity, and BMI), followed by "Genetic/Early Developmental Variables (breastfeeding duration, gestational diabetes, maternal smoking status, birthweight, child age)," and finally "Family Lifestyle Variables (child screen time, sugar sweetened beverage and fruit/vegetable intake, sleep, and preference for vigorous activity; baseline Family Nutrition and Physical Activity scale, adult SF-12 mental scale, and adult 
Table 1. Demographic Information for 450 American Indian Adult-Child Dyads Recruited From 4 Rural Communities and 1 Urban Health Clinic $(n=450)$

\section{Variable}

Parameter

Age (mean [SD])

Adult, y

$31.4(8.45)$

Child, mo

$45.0(12.98)$

Gender, n (\%)

Adult (\% female)

$426(94.7)$

Child (\% female)

$226(50.2)$

Education, $\mathrm{n}(\%)$

High school equivalent or less

169 (37.6)

Some college/associates degree

$235(52.2)$

College degree or postgraduate

$46(10.2)$

Annual household income, $\mathrm{n}(\%)$

$<\$ 5,000$

$132(30)$

$\$ 5,000$ to $\$ 19,999$

$124(28.2)$

$\$ 20,000$ to $\$ 34,999$

$94(21.4)$

$>\$ 35,000$

$90(20.5)$

\begin{tabular}{|c|c|c|}
\hline Participation in Special Supplemental Program for Women, Infants, and Children (\%) & \multicolumn{2}{|c|}{80.9} \\
\hline Food insecure (\%) & \multicolumn{2}{|c|}{61.0} \\
\hline Adults in household (mean, high) & \multicolumn{2}{|c|}{$2.1,8$} \\
\hline Children in household (mean, high) & \multicolumn{2}{|c|}{$2.6,11$} \\
\hline Ethnicity, $n(\%)^{a}$ & Adult & Child \\
\hline American Indian & $368(81.8)$ & $390(86.7)$ \\
\hline Asian & $4(0.9)$ & $6(1.3)$ \\
\hline Native Hawaiian/Pacific Islander & $2(0.4)$ & $2(0.4)$ \\
\hline Black/African American & $4(0.9)$ & $14(3.1)$ \\
\hline Hispanic/Latino & $51(11.3)$ & $80(17.8)$ \\
\hline White & $77(17.1)$ & $89(19.8)$ \\
\hline Other & $1(0.4)$ & $2(0.4)$ \\
\hline
\end{tabular}

${ }^{a}$ Ethnicity percentages total to $>100$ owing to multiple choices.

Perceived Stress Scale scores)" in the third stage. Variables were chosen that are known to be relevant to obesity. Significance was set at $P<.05$.

\section{RESULTS}

\section{Participants}

Table 1 provides sociodemographic information for participants. The study recruited 210 urban-based (46.6\%) and 240 reservation-based dyads (53.4\%). Of the caregivers, 91.1\% were a biological parent (392 mothers and 18 fathers), $7.3 \%$ were a biological grandparent, and $1.6 \%$ identified as aunt or foster parent. Compared with reservation-based participants, urban-based participants had lower income levels $(P=.008)$ and more children were identified as non$\mathrm{AI}(44.7 \%$ vs $29.1 \% ; P=.001)$. Twice as many urban families as those on a reservation consisted of an $\mathrm{AI}$ adult with a non-AI child ( $17 \%$ vs $8 \%)$, most of whom $(57 \%)$ were identified as Hispanic. In the overall sample, $30 \%$ of adults reported total annual income of $<\$ 5,000$ and only $20 \%$ reported
$>\$ 35,000 / y$. Food insecurity was reported for $61 \%$ of households ${ }^{21}$ and $80.9 \%$ of adults reported participation in the Special Supplemental Nutrition Program for Women, Infants, and Children.

\section{Obesity and Related Behaviors}

Table 2 presents descriptive results for the adult BMI and child BMI zscore and health behaviors related to obesity risk. For adults, $60 \%$ were classified as obese, $22 \%$ as overweight, and $18 \%$ as healthy 
Table 2. Cross-sectional Weight Status and Health Behaviors of American Indian Adult-Child Dyads

\section{Variable}

Weight status

Body mass index/body mass index z-score (mean \pm SD)

Healthy weight (\%)

Overweight (\%)

Obese (\%)

Early child development

Gestational diabetes, $\mathrm{n}(\%)$

Maternal smoking during pregnancy, $n$ (\%)

Birth weight, oz (mean \pm SD)

Low birth weight ( $<88 \mathrm{oz}), \mathrm{n}(\%)$

High birth weight (>144 oz), n (\%)

Adult $(n=450)$

Child $(n=450)$

Diet variables in servings/wk (mean $\pm \mathrm{SD}$ )

Sugar-sweetened beverages

Fruits and vegetables

$32.0 \pm 7.9$

18

22

60
$0.78 \pm 1.10$

60

18

22

creen time (all screens: television, phone, computer, video games)

Total daily min (mean \pm SD)

$187.8 \pm 187.3$

$47(11)$

$62(14)$

$118.0 \pm 21.6$

$26(6)$

$30(6)$

Physical activity (mean \pm SD)

15-min bouts of moderate/vigorous activity/wk, $n$

$3.8 \pm 3.8$

Sleep (mean \pm SD), $h$

Weeknight

$8.0 \pm 1.5$

$10.1 \pm 1.1$

Weekend

$8.6 \pm 1.6$

$10.3 \pm 1.1$

Home environment (family nutrition and physical activity) (mean \pm SD)

Total score

$3.08 \pm 0.37$

Nutrition score

$3.22 \pm 0.36$

Physical activity score

$2.91 \pm 0.48$

Readiness to change (mean $\pm \mathrm{SD})^{\mathrm{a}}$

More physical activity

$3.63 \pm 1.06$

More fruits and vegetables

$3.86 \pm 0.90$

Less screen time

$3.04 \pm 1.30$

Less added sugar

$3.71 \pm 1.14$

Manage stress

$3.65 \pm 1.08$

Improve sleep

$3.61 \pm 1.08$

Stress (Perceived Stress Scale) (mean \pm SD)

$16.49 \pm 6.27$

12-Item Short Form Health Survey (mean \pm SD)

Physical health component

$49.18 \pm 8.12$

Mental health component

$46.80 \pm 9.91$

${ }^{a}$ Scale of $1-5$, with higher scores indicating greater readiness to change.

weight. A total of 27 adults were excluded because of current or recent pregnancy. Among children, $22 \%$ were classified as obese, $18 \%$ as overweight, and $60 \%$ as healthy weight. Median adult readiness to change score was 4 (have actively started making changes) on all health behaviors except for screen time, which had a median score of 3 (no, but have been making specific plans to start in the next 30 days). 
Table 3. Factors Predicting Adult Body Mass Index (BMI) and Child BMI z-Score in American Indian Adult-Child Dyads as Determined by Logistic Regression

\section{Predictor}

Predictors of adult BMI

Family nutrition and physical activity nutrition score

Family nutrition and physical activity score

Annual income

Highest education

Food insecurity present

In urban setting

Adult total screen time

Adult fruit and vegetable/d

Adult SSB/wk

SF-12 mental health composite

SF-12 physical health

composite

Adult sleep weekdays

Physical Activity and Nutrition Self-efficacy Scale total score

Perceived Stress Scale total score

Adult is American Indian

Cultural involvement total score

Predictors of child BMI z-score

Sociodemographic variables

$\begin{array}{lr}\text { Highest adult/caregiver } & -.078 \\ \text { education } & \\ \text { Number of adults } & 0.180 \\ \text { Number of children } & -0.033 \\ \text { In urban setting } & -0.141 \\ \text { Income } & -0.078 \\ \text { Adult is American Indian } & 0.279 \\ \text { Food insecurity present } & -0.052 \\ \text { Genetic/early developmental } & \\ \text { variables } & -0.001 \\ \text { Weeks of breastfeeding } & 0.024 \\ \text { Adult BMl } & 0.389 \\ \text { Gestational diabetes present } & 0.265 \\ \text { Mother smoked in pregnancy } & 0.013 \\ \text { Birth weight in oz } & 0.002 \\ \text { Child age in mo } & \end{array}$

B

0.185

0.457

$-0.413$

0.001

0.044

$-0.041$

$-0.008$

$-0.033$

0.094

-0.001
0.024
0.389
0.265
0.013
0.002
95\% Confidence Levels

$\boldsymbol{\beta}$

$\boldsymbol{P}$

-4.335 to 1.312

$-0.067$

$-1.838$

-4.082 to 0.406

$-0.107$

.11

0.103

-0.27 to 0.476

0.037

.59

-0.534 to 0.904

0.033

61

-1.573 to 2.487

0.028

.66

-2.311 to 1.485

$-0.026$

.67

-0.004 to 0.006

0.034

.56

-0.031 to 0.119

0.068

.25

$-0.075$

-0.151 to 0

$-0.119$

.05

-0.161 to 0.078

.50

$-0.273$

-0.387 to -0.158

$-0.053$

$<.001^{\text {** }}$

-0.018 to 0.002

$-0.285$

.14

-0.093 to 0.056

$-0.084$

.62

-0.22 to 0.161

$-0.026$

.74

0.339

-1.692 to 2.37

0.019

.74

-0.294 to 0.482

0.029

.63

$-.078$

-0.164 to 0.008

$-0.105$

.08

0.180

0.061 to 0.299

0.153

$.003^{\text {** }}$

0.116 to 0.050

$-0.042$

.43

0.388 to 0.105

$-0.064$

.26

0.172 to 0.016

$-0.101$

.10

0.039 to 0.519

0.116

$.02^{\star}$

0.302 to 0.198

$-0.023$

.68

0.004 to 0.001

$-0.053$

.31

0.010 to 0.039

0.176

$.001^{\star *}$

0.031 to 0.747

0.110

$.03^{*}$

0.085 to 0.615

0.078

.14

0.008 to 0.019

0.247

$<.001^{\star *}$

0.006 to 0.011

0.026 
Table 3. (Continued)

Predictor

Family lifestyle variables

Child total screen time

Child fruit and vegetable/d

Child SSB/wk

FNPA nutrition score

FNPA activity score

SF-12 mental composite

Child prefers vigorous games

Perceived Stress Scale total score (adult)

Child sleep min, weekdays
B

\begin{tabular}{rl}
\multicolumn{1}{c}{0} & 0.001 to 0.001 \\
-0.052 & 0.136 to 0.031 \\
-0.021 & 0.067 to 0.024 \\
0.208 & 0.173 to 0.588 \\
0.297 & 0.019 to 0.574 \\
0.010 & 0.006 to 0.025 \\
-0.073 & 0.168 to 0.021 \\
0.008 & 0.015 to 0.032
\end{tabular}

95\% Confidence Levels

0.015 to 0.032 $\beta$

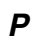

$-0.002 \quad 0.003$ to 0

.78

.22

.36

.28

$.04^{*}$

.22

.13

.49

.07

FNPA indicates family nutrition and physical activity; SF-12, 12-Item Short Form Health Survey; SSB, sugar sweetened beverage.

Note: The data in Table 3 were generated using step-wise regression analysis.

${ }^{\star} P<.05 ;{ }^{\star \star} P<.01$.

Adults reported engaging in moderate to vigorous activity $3.8 \pm 3.8$ times/wk (in 15-minute bouts). The child activity survey does not translate into metabolic equivalents or other comparable measures, so results are not reported here. Families reported that a television was on in the home $5.5 \pm 0.5 \mathrm{~h} / \mathrm{d}$ and that 114 \pm 120 and $92 \pm 87 \mathrm{~min} / \mathrm{d}$ of television were watched for adults and children, respectively. When all screens were included (eg, television, phones, computers, video games, tablets), average watch times were $188 \pm 187 \mathrm{~min} / \mathrm{d}$ for adults and 124 $\pm 120 \mathrm{~min} / \mathrm{d}$ for children. For diet variables, adults consumed $14.5 \pm$ 12.9 servings/wk of sugar-sweetened beverages, compared with $9.2 \pm 9.8$ for children. Combined fruit and vegetable servings per week were $15.9 \pm$ 12.5 and $16.7 \pm 11.9$ for adults and children, respectively.

Adults reported sleeping $8.0 \pm 1.0$ $\mathrm{h} /$ weeknight whereas children slept $10.1 \pm 1.1 \mathrm{~h} /$ weeknight. Adults reported that they fell asleep or went to bed with the television on: never $(58 \%)$, some of the time (22\%), most of the time $(14 \%)$, or always $(6 \%)$. For children, responses were: never (63\%), some of the time (24\%), most of the time $(10 \%)$, or always $(3 \%)$. Adults reported that $51 \%$ of children had a working television in the room where they slept.

\section{Factors Associated With Obesity}

Table 3 provides coefficients for all variables entered into regression models predicting adult BMI and child BMI z-scores. The adult model explained $8.5 \%$ of variance in adult BMI, most of which was attributable to the SF-12 Physical Health Composite score (7.2\% of variance). For children, the overall model explained $16.5 \%$ of variance in the child BMI z-score. For the 3 stages of regression, sociodemographic factors explained $2.6 \%$ of variance, genetic/ early developmental factors $10.5 \%$, and family lifestyle factors $3.4 \%$. Among the sociodemographic factors, higher adult or caregiver education and living in an urban setting were associated with a lower BMI z-score, whereas having a primary caregiver who identified as AI was associated with a higher BMI z-score. Among the genetic and early developmental factors, gestational diabetes, high birth weight, and high adult BMI were positively associated with child BMI zscore. Among the family lifestyle factors, the family nutrition and physical activity total score was associated with an increased child BMI z-score.

\section{DISCUSSION}

This cross-sectional cohort of AI families with young children represented AI communities from extremely rural to urban across 5 states. It was the first cohort of AI families to have included both child and adult data from the same household and information on multiple health habits, including the lesser-studied obesity risk factors of sleep and stress. Novel findings from these data included low child but not adult sleep, high adult readiness to change lifestyle behaviors, and the fact that child BMI z-score was positively predicted by the number of adults in the household and a caregiver who self-identified as AI (among other factors). In addition, adult BMI was predicted by SF-12 physical scores. These data add to the scant literature on obesity risk factors and health behaviors for AI families, who experience a disproportionate risk for obesity.

Similar to many other studies, ${ }^{1,22-24}$ this study found high rates of early childhood overweight and obesity in young AI children (40\%). Multiple behaviors related to obesity risk also were examined, including the first report of sleep data in AI children. Although adults in this sample reported meeting recommended levels of sleep, ${ }^{25}$ these data suggest that a substantial number of AI children may not be meeting current American Academy of Sleep Medicine guidelines. ${ }^{26}$ Daytime nap data were not collected, which represents a limitation of this study. This 
cohort of children had reported screen time use of $\geq 60$ minutes more than are recommended by the American Academy of Pediatrics, ${ }^{27}$ and $>50 \%$ of them had a working television in the room where they slept. The prevalence of low sleep $(<11 \mathrm{~h} /$ night) and high use of screen time $(>2 \mathrm{~h} / \mathrm{d})$ were also seen in 2 other large cohorts of mixed-race (but nonAI) children. ${ }^{28,29}$ A recent longitudinal study ${ }^{30}$ found that inconsistent bedtimes in early childhood predicted obesity in early adolescence but found no relationship between screen time in early childhood and adolescent obesity. The finding that more than half of the children in the current study had a television in their bedroom, however, suggests a possible interaction of these obesity risk factors in this population that warrants further investigation.

Adults in this sample reported underconsuming fruits and vegetables $^{31}$ in conjunction with a high intake of sugar-sweetened beverages (14.5 servings/wk). The younger children in this sample met recommended intake levels of fruits and vegetables, but not the older children. ${ }^{31}$ There was a reported 9.2 servings/wk of sugar-sweetened beverages for child participants overall, which was likely substantially higher than recommended levels of $<10 \%$ added sugars daily. Comparable poor dietary habits were noted for AI children in a South Dakota reservation-based study, ${ }^{22}$ and dietary patterns are known to become less optimal over time in children. $^{32}$ Adults in the current study also reported levels of physical activity approximately 100 minutes below national guidelines of $150 \mathrm{~min} / \mathrm{wk}^{33}$ These findings suggest that a focus on diet and activity in the context of obesity prevention remains appropriate. Moreover, adults in the current study reported high levels of willingness to change diet, activity, and other health behaviors. Some studies found that higher levels of willingness to change are associated with significant levels of subsequent behavior change, ${ }^{34-36}$ which suggests that this variable may be important in understanding health behavior.

This study was unique in providing data on a significant number of urban AI families, who often are not included in large federal-level surveys or any smaller, reservation-based studies. Of note, the research group previously found differences in food insecurity and dietary patterns between urban- and rural-based families in this sample; ${ }^{21}$ similar findings were reported for the general US population. ${ }^{37}$ No significant differences were observed in the other measured health behaviors (eg, adult and child sleep, adult physical activity) in urban vs rural families (data not shown). Although the number of urban and rural families was approximately equal in this sample, only 1 urban-based site was included, which limits the generalizability of the finding to other urban AI families. In addition, the 4 rural reservationbased sites may not be representative of all AI communities nationwide.

Similar to other studies, child BMI z-score was shown to be predicted by family lifestyle variables (family activity) and genetic and early developmental factors (adult BMI, report of gestational diabetes mellitus, high birth weight). ${ }^{38,39}$ However, a positive association of child BMI z-score with maternal smoking in pregnancy was not observed, as was shown in previous studies in Indigenous children, ${ }^{39,40}$ perhaps because maternal smoking rates were much lower in the current sample ( $14 \%$ vs $>40 \%$ in other studies). Interestingly, the number of adults in the household and having a caregiver who identified as AI were positively associated with child BMI z-score. Household number was inversely associated with child BMI in Colombian children, which may be related to food scarcity. ${ }^{41}$ A high prevalence of reported household food insecurity was observed among the families in this sample, but it is unclear how this factor may be affecting child BMI. Moreover, discussions with community partners revealed that a higher number of adults in the household makes lifestyle changes more challenging owing to disruptions in meal and bedtimes, irregular caregiving arrangements, and the increased number of people supported by the household budget and resources. The high number of adults in households reported by the families in the current study is likely related to the high cost and low availability of housing for AI families. A more comprehensive understanding of the interaction of these factors and their contribution to child health is warranted.

This study's data are limited because they were a baseline sample of families who signed up for a 2-year health and safety intervention; therefore, there may have been more motivated families in this cohort. However, in several of the reservation sites, $>60 \%$ of the total number of families with children in this age range were recruited, which suggests that a wider range of families may be represented. Although a range of communities was included, these findings may not be representative of all AI communities. Other limitations include the large number of self-reported measures used and the lack of other sampling strategies for many lifestyle variables. However, the study worked to maximize the accuracy of reported information in 2 ways: local site coordinators assisted adults as they filled out surveys, and several surveys included similar questions. Finally, it is likely that other important obesity predictors were not included in the study. Specifically, household substance abuse, domestic violence, and maternal depression and other factors related to adverse childhood experiences and trauma may influence child weight and represent important areas of future inquiry.

\section{IMPLICATIONS FOR RESEARCH AND PRACTICE}

A more comprehensive understanding of the individual-, family-, and community-level factors influencing obesity is vital to health promotion efforts ongoing in many AI communities. The current findings illustrate the interaction of multiple factors influencing early childhood obesity, including complex family- and environmental-level variables. Future approaches should seek to understand the impact of additional adults in the household and food insecurity on family eating habits and their ability to make nutrition-related 
changes. The study also found inadequate sleep in the children and high levels of caregiver stress, perhaps relating to the high levels of food insecurity, poverty, and historic and intergenerational trauma often experienced by these families. These challenges highlight areas for future intervention and warrant an investigation into the resiliency employed by families to overcome these challenges.

\section{ACKNOWLEDGMENTS}

This study was funded by National Heart, Lung, and Blood Institute Grants R01HL114912 to AKA and T32DK007665 to EJT. The authors gratefully acknowledge all of the communities and families who participated in the design, development, and implementation of the Healthy Children, Strong Families intervention. They are also indebted to the site coordinators who worked so hard to recruit and retain participants.

\section{REFERENCES}

1. Dalenius K BE, Smith B, Polhamus B, Grummer-Strawn L. Pediatric Nutrition Surveillance 2010 Report. Atlanta, GA: US Department of Health and Human Services, Centers for Disease Control and Prevention; 2012.

2. Sharma AJ, Grummer-Strawn LM, Dalenius K, et al. Obesity prevalence among low-income, preschool-aged children-United States, 1998-2008. MMR Morb Mortal Wkly Rep. 2009;58:769-773.

3. Lillie-Blanton M, Roubideaux Y. Understanding and addressing the health care needs of American Indians and Alaska Natives. Am J Public Health. 2005;95:759-761.

4. Castor ML, Smyser MS, Taualii MM, Park AN, Lawson SA, Forquera RA. A nationwide population-based study identifying health disparities between American Indians/Alaska Natives and the general populations living in select urban counties. Am J Public Health. 2006;96:1478-1484.

5. Berns RM, Tomayko EJ, Cronin KA, Prince RJ, Parker T, Adams AK. Development of a culturally informed child safety curriculum for American Indian families. $J$ Prim Prev. 2017;38:195-205.
6. Tomayko EJ, Prince RJ, Cronin KA, et al. Healthy Children, Strong Families 2: a randomized controlled trial of a healthy lifestyle intervention for American Indian families designed using community-based approaches. Clin Trials. 2017;14:152-161.

7. Kuczmarski RJ, Ogden CL, Grummer LM, et al. CDC growth charts: United States. Adv Data. 2000;314:1-27.

8. Division of Nutrition, Physical Activity, and Obesity, National Centers for Chronic Disease Prevention and Health Promotion. Defining adult overweight and obesity. https://www.cdc.gov/ obesity/adult/defining.html. Accessed August 11, 2018.

9. Katula JA, Vitolins MZ, Morgan TM, et al. The Healthy Living Partnerships to Prevent Diabetes study: 2-year outcomes of a randomized controlled trial. Am J Prev Med. 2013;44(suppl 4):S324S332.

10. Eaton DK, Olsen EO, Brener ND, et al. A comparison of fruit and vegetable intake estimates from three survey question sets to estimates from 24-hour dietary recall interviews. I Acad Nutr Diet. 2013;113:1165-1174.

11. Amireault S, Godin G. The GodinShephard leisure-time physical activity questionnaire: validity evidence supporting its use for classifying healthy adults into active and insufficiently active categories. Percept Mot Skills. 2015;120:604-622.

12. Janz KF, Broffitt B, Levy SM. Validation evidence for the Netherlands physical activity questionnaire for young children: the Iowa bone development study. Res Q Exerc Sport. 2005;76:363369.

13. Ihmels MA, Welk GJ, Eisenmann JC, Nusser SM. Development and preliminary validation of a Family Nutrition and Physical Activity (FNPA) screening tool. Int $J$ Behav Nutr Phys Act. 2009;6:14.

14. Hager ER, Quigg AM, Black MM, et al. Development and validity of a 2-item screen to identify families at risk for food insecurity. Pediatrics. 2010;126:e26-e32.

15. Latimer L, Walker LO, Kim S, Pasch KE, Sterling BS. Self-efficacy scale for weight loss among multi-ethnic women of lower income: a psychometric evaluation. J Nutr Educ Behav. 2011;43:279283.

16. Cohen S, Kamarck T, Mermelstein R. A global measure of perceived stress. $J$ Health Soc Behav. 1983;24:385-396.
17. Ware Jr J, Kosinski M, Keller SD. A 12item short-form health survey: construction of scales and preliminary tests of reliability and validity. Med Care. 1996;34:220-233

18. Goins RT, Spencer SM, McGuire LC, Goldberg J, Wen Y, Henderson JA. Adult caregiving among American Indians: the role of cultural factors. Gerontologist. 2011;51:310-320.

19. Palmer PB, O'Connell DG. Regression analysis for prediction: understanding the process. Cardiopulm Phys Ther J. 2009;20:23-26.

20. Pedhazur E. Multiple Regression in Behavioral Research. 3rd ed. Fort Worth, TX: Harcourt Brace College; 1997.

21. Tomayko EJ, Mosso KL, Cronin KA, et al. Household food insecurity and dietary patterns in rural and urban American Indian families with young children. BMC Public Health. 2017;17:611.

22. Story M, Hannan PJ, Fulkerson JA, et al. Bright Start: Description and main outcomes from a group-randomized obesity prevention trial in American Indian children. Obesity (Silver Spring). 2012;20:2241-2249.

23. Adams A, Prince R. Correlates of physical activity in young American Indian children: lessons learned from the Wisconsin Nutrition and Growth Study. J Public Health Manag Pract. 2010;16:394-400.

24. Davis SM, Myers OB, Cruz TH, et al. CHILE: outcomes of a group randomized controlled trial of an intervention to prevent obesity in preschool Hispanic and American Indian children. Prev Med. 2016;89: 162-168.

25. Watson NF, Badr MS, Belenky G, et al. Recommended amount of sleep for a healthy adult: a joint consensus statement of the American Academy of Sleep Medicine and Sleep Research Society. Sleep. 2015;38:843844.

26. Hirshkowitz M, Whiton K, Albert SM, et al. National Sleep Foundation's sleep time duration recommendations: methodology and results summary. Sleep Health. 2015;1:40-43.

27. Council on Communications and Media. Media and young minds. Pediatrics. 2016;138(5).

28. Taveras EM, McDonald J, O'Brien A, et al. Healthy Habits, Happy Homes: methods and baseline data 
of a randomized controlled trial to improve household routines for obesity prevention. Prev Med. 2012;55:418-426.

29. Quick V, Martin-Biggers J, Povis GA, Hongu N, Worobey J, Byrd-Bredbenner C. A socio-ecological examination of weight-related characteristics of the home environment and lifestyles of households with young children. Nutrients. 2017;9(6).

30. Anderson SE, Sacker A, Whitaker RC, Kelly Y. Self-regulation and household routines at age three and obesity at age eleven: longitudinal analysis of the UK Millennium Cohort Study. Int $J$ Obes (Lond). 2017;41:1459-1466.

31. US Department of Health and Human Services and US Dept of Agriculture. 2015-2020 Dietary Guidelines for Americans. 8th ed. http://health.gov/ dietaryguidelines/2015/guidelines/.

Accessed August 11, 2018.

32. Banfield EC, Liu Y, Davis JS, Chang S, Frazier-Wood AC. Poor adherence to US dietary guidelines for children and adolescents in the National Health and Nutrition Examination Survey population. J Acad Nutr Diet. 2016;116:21-27

33. Physical Activity Guidelines Advisory Committee report, 2008. To the Secretary of Health and Human Services. Part A: executive summary. Nutr Rev. 2009;67:114-120.

34. De Nooijer J, Van Assema P, De Vet E, Brug J. How stable are stages of change for nutrition behaviors in the Netherlands. Health Promot Int. 2005;20:27-32.

35. Guess N, Vasantharajah L, Gulliford M, et al. Improvements in stage of change correlate to changes in dietary intake and clinical outcomes in a 5-year lifestyle intervention in young high-risk Sri Lankans. Prev Med. 2016;90:193-200.

36. Mastellos N, Gunn LH, Felix LM, Car J, Majeed A. Transtheoretical model stages of change for dietary and physical exercise modification in weight loss management for overweight and obese adults. Cochrane Database Syst Rev. 2014;2:CD008066.
37. Coleman-Jensen A, Rabbitt MP, Gregory AA, Singh A. Household food security in the United States in 2015. ERR-215. Washington, DC: US Department of Agriculture, Economic Research Service. 2016.

38. Adams AK, Harvey HE, Prince RJ. Association of maternal smoking with overweight at age $3 \mathrm{y}$ in American Indian children. Am J Clin Nutr. 2005;82:393-398.

39. Lindberg SM, Adams AK, Prince RJ. Early predictors of obesity and cardiovascular risk among American Indian children. Matern Child Health J. 2012;16:1879-1886.

40. Thurber KA, Dobbins T, Kirk M, Dance P, Banwell C. Early life predictors of increased body mass index among Indigenous Australian children. PLoS One. 2015;10:e0130039.

41. Gonzalez-Casanova I, Sarmiento OL, Pratt $M$, et al. Individual, family, and community predictors of overweight and obesity among colombian children and adolescents. Prev Chronic Dis. 2014;11:E134.

\title{
Learn About SNEB Position
}

\section{Papers-check out our policy page}

\author{
https://www.jneb.org/content/policy_position_papers
}

